\section{La investigación cualitativa: un aporte para mejorar los servicios de salud}

\author{
PAULA BEDREGAL ${ }^{1}$, CAROLINA BESOAIN ${ }^{2, a}$, \\ ALEJANDRO REINOSO ${ }^{3, b}$, TAMARA ZUBAREW ${ }^{4}$
}

\section{Qualitative research methodology in health care}

Health care research requires different methodological approaches such as qualitative and quantitative analyzes to understand the phenomena under study. Qualitative research is usually the least considered. Central elements of the qualitative method are that the object of study is constituted by perceptions, emotions and beliefs, non-random sampling by purpose, circular process of knowledge construction, and methodological rigor throughout the research process, from quality design to the consistency of results. The objective of this work is to contribute to the methodological knowledge about qualitative research in health services, based on the implementation of the study, "The transition process from pediatric to adult services: perspectives from adolescents with chronic diseases, caregivers and health professionals". The information gathered through the qualitative methodology facilitated the understanding of critical points, barriers and facilitators of the transition process of adolescents with chronic diseases, considering the perspective of users and the health team. This study allowed the design of a transition services model from pediatric to adult health services based on the needs of adolescents with chronic diseases, their caregivers and the health team.

(Rev Med Chile 2017; 145: 373-379)

Key words: Adolescent; Adolescent Health Services; Qualitative Research; Transition to Adult Care.
'Departamento de Salud Pública, Facultad de Medicina, Pontificia Universidad Católica de Chile. Santiago, Chile.

2Escuela de Psicología, Facultad de Psicología, Universidad Alberto Hurtado. Santiago, Chile. ${ }^{3}$ Escuela de Psicología. Facultad de Ciencias Sociales. Pontificia Universidad Católica de Chile.

Santiago, Chile.

${ }^{4}$ División de Pediatría, Facultad de Medicina, Pontificia Universidad Católica de Chile. Santiago, Chile. apsicóloga. PhD en Psicología. bPsicólogo. PhD en Ciencias Sociales.

Financiamiento: FONIS 2013 SA13120067, VRI PUC No $12 / 2013$

Recibido el 17 de junio de 2016, aceptado el 3 de marzo de 2017.

Correspondencia a: Tamara Zubarew

Diagonal Paraguay 362, $8^{\circ}$ Piso.

Santiago, Chile.

tzubarew@gmail.com
$\mathrm{E}$ 1 diseño de nuevos servicios clínicos y su evaluación es parte del quehacer cotidiano de los profesionales de salud. Los métodos de investigación más utilizadas en este ámbito son los llamados mixtos ${ }^{1}$. Los métodos mixtos permiten responder a preguntas de investigación complejas, que abordan aspectos cuantitativos y cualitativos sobre tópicos de interés en salud. El enfoque cuantitativo permite caracterizar los servicios de salud: la cobertura, el acceso, el uso, la efectividad o eficacia de estos y los factores asociados a ello. El enfoque cualitativo permite conocer, desde la perspectiva de los usuarios externos (pacientes y familias o cuidadores) o usuarios internos (profesionales, técnicos, per- sonal de apoyo) las creencias, valores, actitudes, percepciones, opiniones, y modos de vivenciar y actuar respecto de los servicios de salud. Ambos enfoques son complementarios, puesto que entregan información que apunta a diferentes aspectos de la provisión de servicios de salud.

La investigación cualitativa en servicios clínicos es cada vez más frecuente ${ }^{2-4}$. En Estados Unidos de Norteamérica (EEUU), por ejemplo, ha habido un fortalecimiento de esta aproximación metodológi$\mathrm{ca}$ a partir de fondos especiales entregados por el Instituto Nacional de Salud (NIH) $)^{5}$. En Chile no hay incentivos hacia la investigación cualitativa y suele ser especialmente desconocido el diseño e implementación de este tipo de estudio. A di- 
ferencia de lo que sucede en las ciencias sociales, la investigación cualitativa en medicina tiende a ser devaluada y comparada con los alcances y objetivos de los métodos cuantitativos.

El objetivo en este trabajo es contribuir al conocimiento sobre la investigación cualitativa en servicios de salud, mediante la descripción de la implementación de una investigación cualitativa: "El proceso de transición de servicios pediátricos a adultos: visión de adolescentes portadores de enfermedades crónicas, sus cuidadores y los profesionales de salud", cuyo propósito es diseñar un modelo de programas y servicios de transición para adolescentes con enfermedades crónicas, considerando la perspectiva de usuarios y del equipo de salud.

\section{La investigación cualitativa en servicios de salud}

El método cualitativo es relevante cuando investigamos fenómenos sociales complejos que son difíciles de capturar numéricamente, como la red de relaciones que se establece en un servicio clínico ${ }^{6}$. Permite comprender en profundidad el problema, cuáles podrían ser sus causas desde la mirada de los actores sociales, permitiendo -junto con los métodos cuantitativos- desarrollar modelos explicativos y de atención en salud considerando perspectivas personales. También son útiles en la construcción de instrumentos de medición adaptados a las poblaciones.

Las fases metodológicas son similares a la investigación cuantitativa: definir el diseño, la población y muestra, los aspectos a identificar, el tipo de dato a producir (verbal, escritos o visual), el método de producción de los datos (entrevistas individuales o grupales, documentales y la observación), el enfoque analítico a utilizar para analizar los datos y generar conclusiones.

No obstante dichas similitudes, la investigación cualitativa tiene sus propias características. Los objetos de estudio suelen ser las opiniones, conductas, actitudes, creencias, percepciones, emociones de las personas, frente a problemáticas relacionadas con su salud (en este caso, los servicios). Es un proceso orientado a la comprensión desde una lógica principalmente inductiva. Esto implica que el conocimiento es un emergente que no responde a la verificación de hipótesis sino más bien al descubrimiento. Asimismo, el diseño es flexible; es decir, puede ir experimentando modificaciones a lo largo del proceso de investigación, como respuesta a esos mismos emergentes. El objeto de estudio se va dando a conocer a lo largo del proceso y el diseño debe ser lo suficientemente dúctil como para acomodarse a sus características. El diseño cualitativo suele ser circular, justamente para responder a esa flexibilidad. Esto significa que no se recorren sus fases de manera lineal sino en etapas simultáneas sucesivas de muestreo-producción de datos-análisis de datos, lo que permite ir introduciendo ajustes mientras se aprende del problema de investigación ${ }^{7}$.

El diseño de la muestra es central en la investigación cualitativa. En el caso de la investigación cualitativa, el énfasis no está puesto en la representación poblacional ni en la verificación de hipótesis estadísticas, que obligan a utilizar muestras aleatorias, representativas y con potencia estadística que permita el uso de técnicas inferenciales. El énfasis está puesto en la representación teórica y social del objeto de estudio, por lo cual es fundamental la representación que cada persona impone (su contexto, su situación particular). De este modo, el tipo de muestra preferente suele ser no aleatoria, por propósito, puesto que buscamos a sujetos que representen su contexto y nos aporten en la comprensión del objeto. En algunas ocasiones se requerirá, en este proceso circular de construcción de conocimiento, utilizar un tipo de muestreo por propósito: la muestra teórica. Este muestreo implica iniciar con una muestra de arranque, que se origina de los supuestos de representación de los sujetos, pero que dado los análisis preliminares, se aumentarán sujetos hasta lograr que ellos no expresen nada nuevo respecto del objeto. Esto se denomina saturación ${ }^{8,9}$.

El análisis de los datos puede estar orientado a la subjetividad (análisis de contenido, temático, fenomenológico, narrativo y teoría fundamentada), a la descripción de la construcción de las situaciones sociales (análisis conversacional y análisis crítico del discurso $)^{7}$ y a la crítica y cambio social (como por ejemplo: en la investigación acción, y algunas perspectivas feministas) ${ }^{10}$.

Otro de los aspectos centrales en la investigación cualitativa es el rigor. En ciencia existe consenso en que es necesario tener criterios de calidad para la investigación cuantitativa y cualitativa. Sin embargo, existe una diversidad de enfoques respecto a cómo asegurarlos en la investigación cua- 
Investigación cualitativa en salud - P. Bedregal et al

Tabla 1. Criterios de rigor y equivalencia entre aproximaciones metodológicas

\begin{tabular}{|c|c|c|}
\hline Enfoque cuantitativo & Enfoque cualitativo & Observaciones \\
\hline Adecuación epistemológica & Adecuación epistemológica & $\begin{array}{l}\text { La pregunta de investigación determina si el método seguido } \\
\text { es coherente con el paradigma. El análisis de datos se relacio- } \\
\text { na con lo que se indaga }\end{array}$ \\
\hline Relevancia & Relevancia & La pregunta aporta al conocimiento científico o técnico \\
\hline \multirow[t]{2}{*}{ Validez interna } & Credibilidad & $\begin{array}{l}\text { El método es riguroso y coherente } \\
\text { Los investigadores son creíbles } \\
\text { La investigación es científica }\end{array}$ \\
\hline & Reproducible & El diseño es replicable \\
\hline \multirow[t]{2}{*}{ Generalización estadística } & Transferencia & Depende del contexto \\
\hline & Generalización teórica & Nutre a la teoría, la mejora \\
\hline \multirow[t]{2}{*}{ Confiabilidad } & Confiabilidad & Consistencia entre pregunta-paradigma y análisis propuesto \\
\hline & Reflexividad & $\begin{array}{l}\text { Autocrítica del papel del investigador en el proceso de in- } \\
\text { vestigación }\end{array}$ \\
\hline
\end{tabular}

litativa, de manera de tomar en consideración las diferencias epistemológicas y las particularidades de las diferentes disciplinas ${ }^{11}$. Una salida posible desde la investigación en salud es intentar una equivalencia aproximada a los conceptos de rigor usados en los métodos cuantitativos (Tabla 1). La credibilidad del estudio está determinada por múltiples aspectos que incluyen desde la calidad del diseño hasta la coherencia de los resultados. Un aspecto interesante en el análisis cualitativo es la dependencia, que se refiere al grado en que los investigadores dan cuenta o describen los contextos cambiantes y las circunstancias observadas durante la ejecución del estudio, que afectan la validez interna del estudio. En términos de generalización, existen dos alternativas: la transferencia, que es el grado en que los temas que emergen del estudio pueden ser transferidos a otro contexto, o la generalización teórica, que es en qué medida los datos me permiten construir o aportar en la construcción de una nueva teoría ${ }^{12}$.

\section{Una experiencia: la investigación sobre transición de jóvenes portadores de enfermedades crónicas}

Nuestra investigación buscó comprender el proceso de transición del cuidado pediátrico al de adultos, desde los adolescentes portadores de enfermedades crónicas, sus cuidadores y los profesionales de salud que participan en este.
Se propuso un estudio de carácter analítico-relacional, de carácter cualitativo, basado en las orientaciones analíticas de la Teoría Fundamentada ${ }^{12,13}$. Para identificar a los participantes, se realizó un muestreo por propósito ${ }^{13}$, utilizando los criterios de enfermedad crónica descrito por Stein ${ }^{14}$. La muestra por propósito fue de 84 participantes fue organizada en tres grupos:

\section{Adolescentes}

Treinta y dos jóvenes de 15 a 24 años, portadores de enfermedades crónicas que cumplen con los requisitos de Stein ${ }^{14}$, próximos a experimentar o han experimentado la transición entre los servicios pediátricos y de adultos en la Red de Salud UC-Christus y Hospital Dr. Sótero del Río, Servicio de Salud Metropolitano Sur Oriente, Santiago, Chile. Estos tenían al menos dos años de evolución de su enfermedad y portaban enfermedades que pueden comprometer gravemente la vida y requieren asistencia médica intensa y contacto frecuente con los servicios de salud.

\section{Cuidadores}

Treinta y dos madres, padres u otros familiares que estén a cargo del cuidado de adolescentes con enfermedad crónica.

\section{Profesionales de salud}

Veinte profesionales de salud (15 médicos, 3 enfermeras y 2 psicólogos), 10 de ellos de servicios 
pediátricos y 10 de adultos. Tanto adolescentes como cuidadores y profesionales de salud provenían en cantidades equivalentes de ambos centros de salud.

A continuación se detalla el modo específico en que estos lineamientos generales fueron implementados en esta investigación particular, lo cual nos permitirá luego discutir los rendimientos que la investigación cualitativa puede tener para la investigación en salud.

\section{La base de un estudio cualitativo: un equipo plural}

Un aspecto fundamental del proceso de investigación cualitativo fue la conformación del equipo de investigación: médicos, enfermeras y psicólogos con diversidad de trayectorias profesionales y de investigación. Esta diversidad permitió dar mayor rigor a todo el proceso investigativo, desde la producción de datos hasta el análisis, siguiendo las propuestas de Denzin ${ }^{15}$ y Cornejo, Besoain y Mendoza ${ }^{16}$ relativas a la triangulación como criterio de rigor metodológico. Se trata de dar rigor al proceso de investigación cualitativo a partir de la inclusión de una diversidad de miradas y ópticas hacia el objeto de estudio, que permita una contraposición y comparación entre perspectivas, lo cual otorga densidad a los análisis. En particular, la diversidad disciplinaria, generacional y de género de nuestro equipo fue una apuesta metodológica que permitió ampliar los márgenes de la reflexión, haciendo parte de la escena investigativa con actores estrechamente vinculados al objeto de estudio (como fue el caso de los investigadores vinculados a medicina) y actores más distantes del fenómeno, pero con formación en las metodologías y los procesos adolescentes (como fue el caso de los psicólogos y psicólogas miembros del equipo).

\section{Dispositivos metodológicos reflexivos}

Bajo sus diversas formas o condiciones, el encuentro entre investigador y participante en la investigación cualitativa constituye una práctica interpretativa, activa, reflexiva y dialogada ${ }^{16}$. Desde la epistemología cualitativa los datos son co-creados en el diálogo. Tal como señalara Denzin ${ }^{15}$, en este encuentro se producen textos o palabras que tienen un carácter performativo, en la medida que traen a escena los mundos del investigador y el participante. Así, el encuentro entre investiga- dor y participante es una práctica reflexiva entre dos actores que crean un mundo, donde todas sus dimensiones deben ser objeto de interpretación. La entrevista transcrita es inseparable de las condiciones bajo las cuales fue producida y su consideración es indispensable para comprender adecuadamente los resultados.

Lo anterior otorga a los procesos de producción y análisis de datos cualitativos un carácter particular. La investigación cualitativa ha compartido como característica distintiva la consideración de la subjetividad como característica del saber $y$ del pensar científico ${ }^{17}$. Esto significa que todo conocimiento está inevitablemente afectado por las características del investigador. Para enfrentar la pregunta sobre cómo traducir a herramientas metodológicas la consideraciones de la subjetividad del investigador y ponerla al servicio de la investigación, diversos autores, tales como Russel \& Kelly ${ }^{18}$ y Cornejo, Besoain y Mendoza ${ }^{16}$, coinciden en que la clave está en la reflexividad. Esta ha sido definida como un ejercicio constante del equipo de investigación orientado a conseguir un entendimiento amplio y lúcido del proceso a través del cual se lleva a cabo la investigación. Se trata de volver al investigador y su actividad como objeto de análisis, de modo de desarrollar una conciencia crítica respecto de la acción del investigador.

En nuestra investigación generamos un dispositivo metodológico atento a estas consideraciones sobre la reflexividad, desde la producción de datos hasta las fases de análisis y escritura. La producción de datos fue realizada en etapas. En un primer momento se realizó una etapa piloto, que contempló la realización de entrevistas a dos adolescentes y dos cuidadores, provenientes de ambos centros de salud. Tras la realización de un análisis preliminar de las entrevistas se ajustaron los guiones de entrevista. En un segundo momento se realizó el resto de las entrevistas en tres subequipos simultáneos -de adolescentes, padres y profesionales de la salud- en ambos centros de salud. Cada subequipo se reunió quincenalmente para discutir ajustes a los guiones de entrevista, evaluar que el material producido fuera dando cuenta del fenómeno de interés y realizar análisis preliminares. La realización de estas entrevistas fue acompañada por la implementación de "dispositivos de escucha" 16 , esto es, dispositivos para el registro de aquello que no queda contenido en la transcripción de la entrevista (aspectos no ver- 
bales, dimensión emocional tanto del participante como del investigador, primeras impresiones sobre la entrevista). Estos fueron llamados, siguiendo la tradición etnográfica, Cuadernos Reflexivos. Este material fue incluido en los análisis de cada subequipo y contribuyó a los procesos de codificación abierta y axial.

El método de comparación continua propuesto por la Teoría Fundamentada incluye procedimientos sistemáticos y explícitos de codificación y análisis que permiten hacer plausibles diferentes categorías, propiedades e hipótesis comprensivas emergentes acerca del fenómeno en estudio. Se realizó en una primera etapa una codificación abierta de las entrevistas, lo que permitió la construcción de tres sistemas de códigos para las entrevistas a adolescentes, a profesionales y a cuidadores.

Los avances de cada subequipo fueron compartidos y supervisados en reuniones mensuales de interanálisis ${ }^{16,19}$, en las cuales se sometían a discusión las categorías de cada subequipo. Los procesos de producción de entrevistas fueron simultáneos a los procesos de análisis de cada subequipo y de interanálisis de equipo general. Tal como señala Creswell ${ }^{20}$, el análisis de datos fue circular. Así, la producción y el análisis se realizaron en fases integradas y simultáneas, ofreciendo pistas analíticas que permitieron guiar el muestreo (a quién entrevistar en qué momento) y ajustar los guiones de entrevista atendiendo los emergentes del trabajo de campo.

\section{Consideraciones éticas}

En investigación cualitativa, la ética es una dimensión especialmente sensible, justamente porque la investigación se sostiene en el encuentro entre investigador y participante, y es el diálogo entre ambos lo que permite la construcción de los resultados. Desde esta perspectiva, los aspectos éticos de una investigación remiten tanto al aseguramiento de la voluntariedad de participación, la confidencialidad y trato cuidadoso de la información, como también a la atención a la dimensión ética del conocimiento.

Respecto de lo primero, esta investigación implicó varios desafíos. Por una parte, los procesos de reclutamiento de los adolescentes y cuidadores fueron cautos en asegurar la participación voluntaria. Los pacientes fueron identificados por sus enfermeras o médicos tratantes y luego algún miembro del equipo de investigación invitó a los adolescentes y cuidadores a una entrevista. En el caso de las entrevistas a los profesionales de la salud, atendiendo a que se trataba de una investigación entre pares, se decidió que los entrevistadores fuesen siempre psicólogos/as del equipo, de manera de facilitar una conversación fluida, minimizando sentimientos de evaluación o competencia. Antes de comenzar cada una de las entrevistas se dio lugar a la lectura y firma de consentimientos informados, dando espacio para responder dudas o preguntas.

Respecto de la ética del conocimiento, los investigadores se encuentran inevitablemente en una posición de poder que les permite decidir los modos en los que visibilizará y hará sentido de la información. Por ello, es tan importante transparentar las opciones metodológicas tomadas. Este es un gran desafío para la investigación cualitativa, ya que muchas veces, por criterios de los formatos de publicación, la descripción de la metodología es muy breve y omite la voz y criterios del equipo de investigación ${ }^{17}$. El presente artículo intenta ser un aporte en este sentido, al visibilizar lo que por algunos autores ha sido llamado "la trastienda investigativa" ${ }^{21}$.

\section{Síntesis de resultados de la investigación: "Servicios de transición de jóvenes portadores de enfermedades crónicas"}

El análisis de las entrevistas realizadas a jóvenes, cuidadores y profesionales, identificó cinco puntos críticos en la transición de adolescentes portadores de enfermedades crónicas del cuidado pediátrico a servicios de adultos, en que hay consenso entre todos los actores participantes:

1. Existencia de fuerte vínculo entre profesional tratante, adolescente portador de enfermedad crónica y cuidador.

2. Resistencia a la transferencia desde los servicios pediátricos a los de adultos, tanto de adolescentes y cuidadores, como del equipo pediátrico.

3. Dificultad en la coordinación entre el equipo pediátrico y adulto durante la transferencia.

4. Dificultad en el desarrollo de la autonomía del adolescente portador de enfermedad crónica.

5. Invisibilidad de la adolescencia en el proceso de atención de la enfermedad crónica.

De estos puntos críticos se analizaron las condiciones causales, intervinientes, correlatos con- 
ductuales y consecuencias, en base a la propuesta de la Teoría Fundamentada. La información obtenida se estudió a la luz de investigaciones internacionales en el mismo tema ${ }^{22-26}$. Se concluyó de esta investigación la necesidad de implementar un modelo de transición que considere al adolescente con sus características y necesidades particulares, que incluya tanto a la familia como a los profesionales de salud involucrados, y que permita y promueva la comunicación entre los servicios pediátricos y de adultos. Basado en los hallazgos de esta investigación, se plantearon lineamientos para la implementación de procesos graduales y planificados de transición desde los servicios pediátricos a servicios de adultos.

\section{Conclusión}

Los métodos cualitativos en la investigación han adquirido cada vez mayor importancia en la comprensión de las experiencias, procesos y conductas relacionadas a la utilización de servicios de salud. El proceso de implementación requiere considerar los criterios de rigor propios del paradigma y la constitución de equipos entrenados y motivados.

En la investigación, esta metodología fue desarrollada en base a entrevistas semiestructuradas con preguntas directrices abiertas orientadas a los objetivos, en que se dejó fluir el relato de los participantes, obteniendo un amplio rango de respuestas y una comprensión en profundidad de las experiencias, percepciones, procesos y conductas. Esta perspectiva difícilmente habría podido ser obtenida a través de un estudio solamente cuantitativo. La posibilidad de escuchar los relatos desde la propia voz de los participantes, pacientes, cuidadores y profesionales, aportó valiosa información respecto a la comprensión de aspectos críticos de la atención de salud de estos pacientes, sobre el vínculo adolescente-cuidadorprofesional, la resistencia a la transición, el desarrollo de la autonomía en adolescentes portadores de enfermedades crónicas, la invisibilidad de la adolescencia y la dificultad en la coordinación de la atención pediátrica-adultos, a partir de lo cual se diseñaron lineamientos de atención.

De este modo, se constituye en una experiencia investigativa valiosa y aportadora hacia la comprensión de fenómenos sociales en la atención en salud, dando vida a la voz de los actores, para los cuales se elaboran y diseñan políticas, programas y servicios.

\section{Referencias}

1. Hansen M, O’Brien K, Meckle G, Chang AM, Guise J-M. Understanding the value of mixed methods research: the Children's Safety Initiative-Emergency Medical Services. Emerg Med J 2016.

2. Shortell SM. The emergence of qualitative methods in health services research. Health Serv Res 1999; 34: 108390.

3. McKibbon KA, Gadd CS. A quantitative analysis of qualitative studies in clinical journals for the $2000 \mathrm{pu}-$ blishing year. BMC Med Inform Decis Making 2004; 4: 11.

4. Hoff TJ, Witt LC. Exploring the use of qualitative methods in published health services and management research. Med Care Res Rev 2000; 57: 139-60.

5. National Institutes of Health. Methodology and Measurement in the Behavioral and Social Sciences (RO1) Program Announcement. Disponible en: http:/grants. nih.gov/grants/guide/pa-files/PAR-16-261.html (Extraído el 26 de mayo de 2016).

6. Curry L, Nembhard IM, Bradley E. Qualitative and Mixed Methods Provide Unique Contributions to Outcomes Research C irculation 2009; 119: 1442-52.

7. Flick, U. State of the Art. Qualitative Research 2002; 41: 5-24.

8. Sandoval CA. Módulo 4. Investigación cualitativa. Módulos de Investigación Social. Programa de Especialización en Teoría, Métodos y Técnicas de Investigación Social. Bogotá-Colombia: ARFO Editores. 1996.

9. Robinson OC. Sampling in Interview-Based Qualitative Re-search: A Theoretical and Practical Guide, Qualitative Research in Psychology 2014; 11 (1): 25-41.

10. Cornejo M, Salas N. Rigor y Calidad Metodológicos: Un Reto a la Investigación Social Cualitativa. Psicoperspectivas 2011; 10: 12-34.

11. Devers KJ. How will we know "good" qualitative research when we see it? Beginning the dialogue in health services research. Health Serv Res 1999; 34: 1153-88.

12. Strauss A, Corbin J. Bases de la investigación cualitativa. Técnicas y procedimientos para desarrollar la teoría fundamentada. Colombia: Editorial Universidad de Antioquia. 2002.

13. Patton M. Qualitative Research and Evaluation Methods. Thousand Oaks, CA: Sage Publications. 2002.

14. Sawyer SM, Drew S, Yeo MS, Britto MT. Adolescents 
with chronic conditions: challenges living, challenges treating. Lancet 2007; 369: 1481-9.

15. Denzin N. The reflexive interview and perfomative social science. Qualitative Research 2001; 1: 23-46.

16. Cornejo M, Besoain C, Mendoza F. Desafíos en la generación de conocimiento en la investigación social cualitativa contemporánea. Forum Qualitative Sozialforschung / Forum: Qualitative Social Research. 2011: 12: Art. 9.

17. Breuer F. (2003). Lo subjetivo del conocimiento sociocientífico y su reflexión: ventanas epistemológicas y traducciones metodológicas. Forum Qualitative Sozialforschung / Qualitative Social Research 4 (2), Art. 25. Disponible en: http://nbn-resolving.de: 0114fgs0302258.

18. Russell G, Kelly N. Research as interacting dialogic processes: Implications for reflexivity. Forum Qualitative Sozialforschung/Forum: Qualitative Social Research. 2002; 3 Art. 18.

19. Cornejo M, Mendoza F, Rojas R. La investigación con relatos de vida: Pistas y opciones del diseño metodológico. Psykhe 2008; 17: 29-39.

20. Creswell JW. Qualitative inquiry and research design: Choosing among qualitative approaches ( $2 \mathrm{nd}$ ed.). Thousand Oaks, CA: Sage. 2007.
21. Wainerman C, Sautu R. La trastienda de la investigación. Buenos Aires: Lumière. 2001.

22. American Academy of Pediatrics, American Academy of Family Physicians and American College of Physicians-American Society of Internal Medicine. A Consensus Statement on Health Care Transitions for Young Adults with Special Health Care Needs. Pediatrics 2002; 110: 1304-6.

23. Society for Adolescent Medicine. Transition to adult health care for adolescents and young adults with chronic conditions. A position paper. Journal of Adolescent Health 2003; 33: 309-11.

24. Transition from children's to adults' services for young people using health or social care services. NICE guideline. February 2016. Disponible en: www.nice.org.uk/ guidance/ng43 (Extraído el 28 de marzo de 2016).

25. National Center for Health Care Transition Improvement. Six Core Elements of Health Care Transition. January 2014. Disponible en: www.gottransition.org. (Extraído el 28 de marzo de 2016).

26. Australian Physiotherapy Association. Position Statement: Transition of Young people with a chronic health condition to adult health services. 2014. Disponible en: www.physiotherapy.asn.au (Extraído el 28 de marzo de 2016). 